\title{
Association between Family Functionality and Depression: A Systematic Review and Meta-Analysis
}

\author{
Daniel Guerrero-Muñoz', Diana Salazar', Victoria Constain ${ }^{1}$, Alvaro Perez', Carlos Andrés Pineda-Cañar ${ }^{1,2}$, \\ Herney Andrés García-Perdomo ${ }^{2,3, *}$
}

'Department of Family Medicine, Universidad del Valle, Cali, Colombia

${ }^{2}$ School of Medicine, Universidad del Valle, Cali, Colombia

${ }^{3}$ UROGIV Research Group, Universidad del Valle, Cali, Colombia

Background: Different scales have attempted to assess various aspects of family dynamics and structures. Good family function seems to allow for better prognoses for basic diseases and appears to be a predictor of depression. The aim of this study was to determine the association between family functionality and depression.

Methods: This is a systematic review and meta-analysis including cross-sectional, cohort, and case-control studies using validated instruments such as the Family APGAR (Adaptability, Partnership, Growth, Affection, and Resolve) and Family Adaptability and Cohesion Evaluation Scale III. A search strategy was designed for the MEDLINE, Embase, Central, and LILACS databases along with data saturation through a search of unpublished literature from the onset of the databases to the present. The categorical variables are expressed in terms of odds ratios (OR), and the statistical analysis was carried out using Review Manager ver. 5.31 (The Cochrane Collaboration, Oxford, UK) using forest plots with corresponding 95\% confidence intervals (CI). A fixed-effect model was used according to the expected heterogeneity, expressed in terms of $\mathrm{I}^{2}$. The risk of bias was evaluated using the MINORS (methodological index for non-randomized studies) tool.

Results: A total of 1,519 studies were found, of which 10 were selected for the qualitative synthesis and four were chosen for the meta-analysis. The result for the association between family dysfunction and depression yielded an OR (95\% CI) of 3.72 (2.70 to 5.12$)$ and $\mathrm{I}^{2}$ of $24 \%$.

Conclusion: Family dysfunction and depression are strongly associated.

Keywords: Apgar Score; Depression; Family Dysfunction; Meta-Analysis; Systematic Review

Received: December 13, 2019, Revised: February 18, 2020, Accepted: February 28, 2020

*Corresponding Author: Herney Andrés García-Perdomo https://orcid.org/0000-0001-6945-8261

Tel: +57-3212195102, E-mail: Herney.garcia@correounivalle.edu.co 


\section{INTRODUCTION}

In healthcare, the holistic vision of an individual is increasingly important, regardless of whether the approach is from a primary care or hospital services perspective.

There are several levels of knowledge regarding the patient as a person: an individual level with a physical or biological dimension, a psychological level with emotional and intellectual aspects, and a spiritual level. Finally, there is a social level, in which family, community, and cultural aspects stand out. ${ }^{1,2)}$

Several instruments have been developed to assess some of these dimensions. The dimensions most studied and developed in clinical practice are those related to emotions and family. Regarding the former, the emphasis has been on detecting depressive disorders, given the regarding healthcare costs and loss of work-hours. In turn, different scales have attempted to assess aspects of family dynamics and structures, such as the Family APGAR (Adaptability, Partnership, Growth, Affection, and Resolve), which obtains a qualitative measurement of the individual's satisfaction regarding their family's functioning, $^{3,4)}$ the Gijon Social-Familial Evaluation Scale (SFES), which assesses social risk $;{ }^{5)}$ and the Family Adaptability and Cohesion Evaluation Scale III (FACES III), which evaluates family functionality through cohesion and flexibility. ${ }^{6,7)}$

In addition, we know from previous studies ${ }^{4,6,8)}$ that good family function seems to allow for better prognoses for basic diseases and appears to be a predictor of depression, which is a major public health problem that can lead to high functional disability and mortality; it has a lifetime global prevalence of $12 \%{ }^{8)}$ This condition carries an important psychosocial burden in which family support is crucial. Previous studies have shown that family environment variables (structure, func- tion, support, and conflicts, among others) are related to the disease management, adherence to treatment, coping, complications, and the psychological adaptation of the individual and family to different pathologies. ${ }^{3)}$ However, thus far, we lack consistent scientific evidence that allows for the use of these instruments as elements associated with family functionality and patients with depression.

This study therefore aimed to determine the association between family functionality and depression.

\section{METHODS}

The present research followed the quality criteria of the Cochrane Collaboration and was guided by PRISMA (Preferred Reporting Items for Systematic Reviews and Meta-Analyses) standards for performing systematic reviews. The protocol was registered in PROSPERO: CRD4018 106455.

\section{Eligibility Criteria}

We included all available studies (cross-sectional, case-control, and cohort) that used validated instruments to assess family function, such as the Family APGAR, Gijon SFES, FACES III, and clinical criteria in pediatric and adult populations from both genders. There were no language restrictions. The evaluations were carried out in the community or through outpatient or in-hospital consultations.

\section{Exclusion Criteria}

We excluded patients with previous psychiatric diagnoses other than depression.

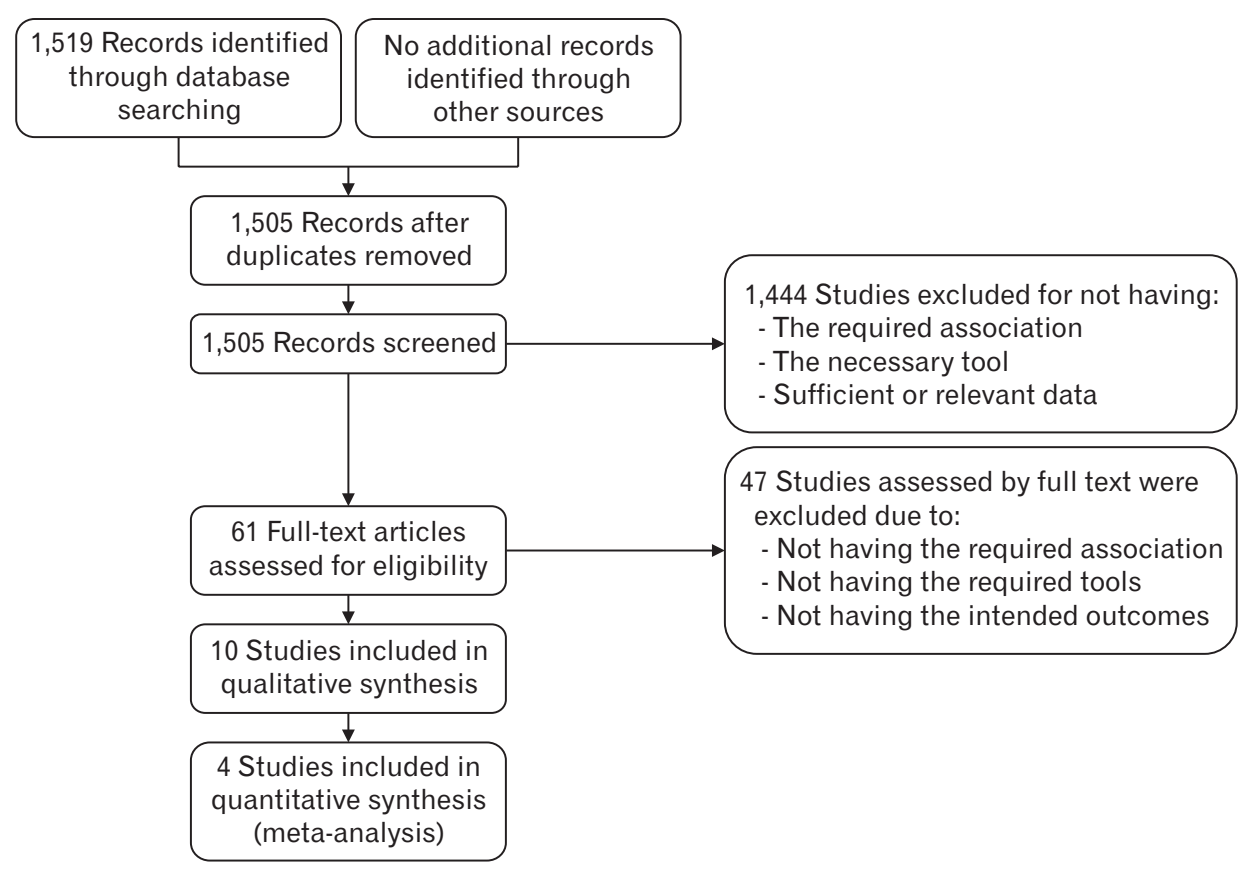

Figure 1. Diagram of included studies. 


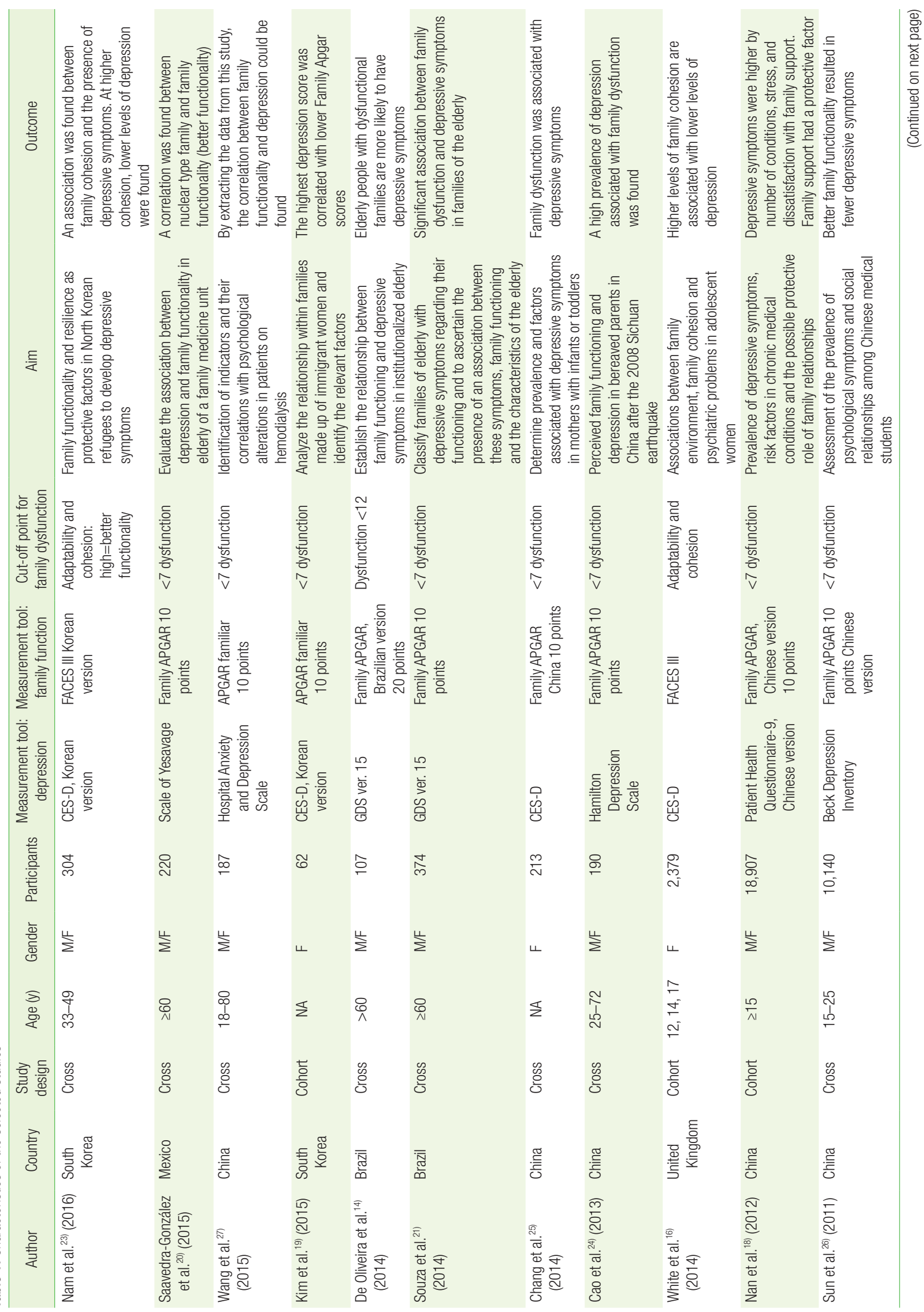




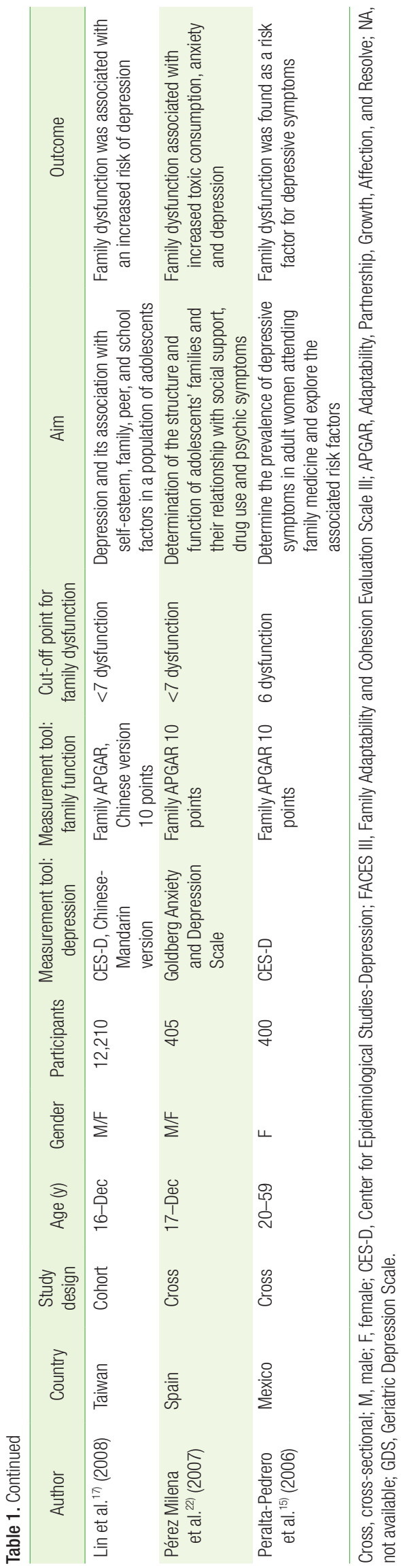

\section{Primary Outcome}

The primary outcome was the association between family functionality and depression. We accepted the definition based on the Diagnostic and Statistical Manual of Mental Disorders of the American Psychiatric Association and the International Classification of Diseases in all their versions. ${ }^{9-11)}$ Likewise, depression screening scales were included.

\section{Data Sources and Search Strategy}

A search strategy was designed for the following databases: MEDLINE (National Library of Medicine, Bethesda, MD, USA), Ovid (Wolters Kluwer, New York, NY, USA), Embase (Elsevier, Amsterdam, Netherlands), and LILACS.

For the data saturation, a generic search strategy was designed for Google Scholar (Google Inc., Mountain View, CA, USA), reference lists, Open Grey, and thesis and seminar databases. There were no restrictions based on language or the statuses of publication of articles. The complete search strategy in each database can be viewed in Appendix 1 .

\section{Selection of Studies}

The researchers performed the initial search according to the proposed strategies. Duplicates were eliminated, and two evaluators assessed the selected studies using the title and abstract, including those that met the criteria. Two evaluators reviewed the full texts obtained, and in cases of disagreement, they discussed the case with a third evaluator. The studies that met the inclusion criteria were subjected to the required quality coding and evaluation.

\section{Data Extraction and Management}

Two evaluators extracted the data from each complete study (after evaluating the inclusion criteria) blindly and independently, and cases of differences were resolved through discussions within the research group.

\section{Risk of Bias Evaluation}

Two researchers assessed the risk of bias independently in and between individual studies using the methodological index for non-randomized studies (MINORS instrument). ${ }^{12)}$ Disagreements were resolved through consensus.

\section{Statistical Analysis and Data Synthesis}

The categorical variables were expressed in terms of odds ratios (OR), and the statistical analysis was carried out using Review Manager ver. 5.31 (The Cochrane Collaboration, Oxford, UK) using forest plots with corresponding 95\% confidence intervals (95\% CIs). A fixed-effect model was used according to the expected heterogeneity. We extracted the adjusted OR from one study and calculated the others ORs based on raw data (three studies).

\section{Heterogeneity}

The heterogeneity was expressed in terms of $\mathrm{I}^{2}$, which was interpreted 
as follows: less than $50 \%$, low heterogeneity; greater than $50 \%$, high heterogeneity. ${ }^{13)}$

\section{Publication Bias}

No evaluation was carried out given the few studies included in the meta-analysis.

A

A clearly stated aim

Inclusion of consecutive patients

Prospective collection of data

Endpoints appropriate to the aim of the study

Unbiased assessment of the study endpoint

Follow-up period appropriate to the aim of the study

Lost to follow up less than $5 \%$

Prospective calculation of the study size

An adequate control group

Contemporary groups

Baseline equivalence of groups

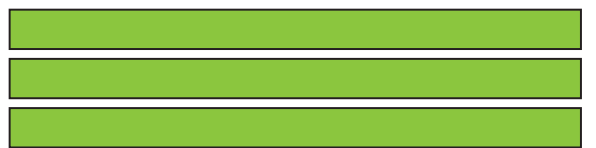

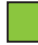

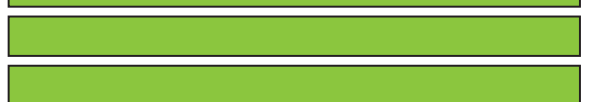

Adequate statistical analyses

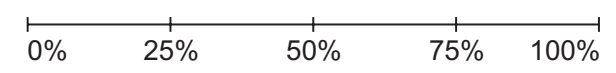

\begin{tabular}{lll}
\hline Low risk of bias $\quad \square$ Unclear risk of bias $\quad \square$ High risk of bias \\
\hline
\end{tabular}

B
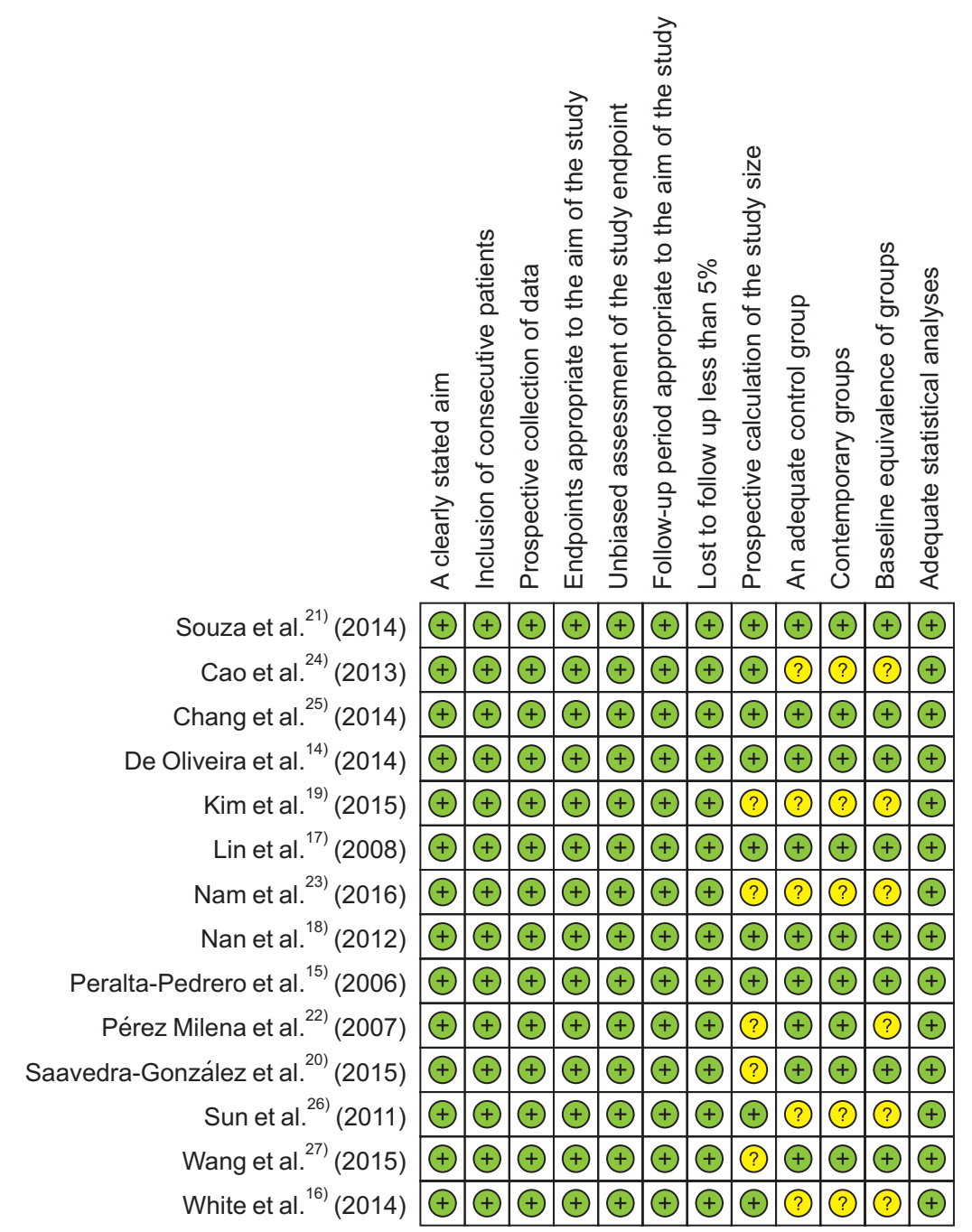

Figure 2. (A) Risks of bias in the studies. (B) Risks of bias within the studies. 


\section{Subgroup Analysis}

A subgroup analysis was proposed, although it was nonviable given the few studies included.

\section{RESULTS}

\section{Selection of Studies}

The search strategy in the databases delivered 1,519 records. After eliminating duplicates and nonrelevant articles according to the inclusion and exclusion criteria, 10 studies were selected for a qualitative analysis and four for a quantitative analysis (Figure 1). ${ }^{14-27)}$

\section{Characteristics of the Selected Studies}

Ten cross-sectional studies ${ }^{14,15,20-27)}$ and four cohort studies ${ }^{16-19)}$ were selected, which accounted for a total of 46,095 patients. The Family APGAR was used in 12 studies as a screening tool for family functionality, while the FACES III was used in two studies. The search yielded no results that used the Gijon SFES.

Regarding depression diagnoses, we found considerable variations in the instruments used. Four studies included a quantitative comparative analysis between family functionality and depression. Table 1 shows the remaining basic characteristics.

\section{Characteristics of Excluded Studies}

Forty-seven references were excluded after reviewing the full texts because they contained no relevant information and/or did not meet the inclusion criteria.

\section{Risk of Bias in Studies}

The 14 selected studies were evaluated according to the MINORS bias risk tool; all the studies had a low risk of bias for the following items: clearly stated aim, consecutive inclusion of patients, appropriate results regarding the aim of the study, appropriate follow-up regarding the aim of the study, losses during follow-up of less than $5 \%$, and adequate statistical analysis. Five studies were found to have an unclear risk of bias for the following items: prospective calculation of the study size, control group, contemporary groups, and equivalence in the basic characteristics of the groups (Figure 2A, B).

\section{Family Functionality and Depression}

Fourteen studies evaluated family function and depression as the primary outcome. However, only four studies had sufficient data to analyze the ORs and the corresponding 95\% CIs to perform a meta-analysis. ${ }^{14,15,20,21)}$ An OR of 3.72 (95\% CI, 2.70 to 5.12; $\mathrm{I}^{2}=24 \%$ ) was found, showing that family dysfunction and depression are associated (Figure 3).

\section{DISCUSSION}

\section{Summary}

Ten studies were selected for a qualitative synthesis and four for a meta-analysis, with an OR of 3.72 (95\% CI, 2.70 to 5.12), low heterogeneity, and a low risk of bias for the selected studies.

\section{Comparison with Existing Literature}

\section{1) Effect of individuals' age and family dysfunction on risk for depression}

Reduced family cohesion, as valued by parents, was associated with psychiatric symptoms in adolescents. Adolescents between 12 and 14 years of age in families with low levels of cohesion and control showed depressive symptoms at a higher frequency. ${ }^{16)}$ Among the adolescent population, women with poor family perceptions were more likely to be diagnosed with depression or have depressive symptoms, with an exponential correlation to greater deterioration of family function. ${ }^{17,22)}$

In institutionalized older adults (OAs), ${ }^{14)}$ family dysfunction has been linked to depressive symptoms. In this context, OAs with familial dysfunction have a 5.36-fold higher risk of experiencing depressive symptoms (95\% CI, 3.03-9.5) than individuals of similar ages with good family function. ${ }^{21)}$ Other variables associated with family functionality may behave as confounding factors when predicting an individual's depression risk, such as the family typology. However, when disaggregating this variable in a study in OAs that initially showed no association, the results obtained were consistent with those of other studies already mentioned for this age group; meaning that family dysfunction, in terms of its typology, was a risk factor for depression regardless of the family structure. ${ }^{20)}$

\begin{tabular}{|c|c|c|c|c|c|c|c|c|}
\hline \multirow{2}{*}{$\begin{array}{l}\text { Study or subgroup } \\
\text { Souza et al. }^{21)}(2014)\end{array}$} & \multicolumn{2}{|c|}{$\begin{array}{l}\text { dysfunction } \\
\text { Events Total }\end{array}$} & \multicolumn{3}{|c|}{ No dysfunction } & $\begin{array}{c}\text { Odds ratio } \\
\text { IV, fixed, } 95 \% \mathrm{Cl}\end{array}$ & \multicolumn{2}{|c|}{$\begin{array}{l}\text { Odds ratio } \\
\text { IV, fixed, } 95 \% \mathrm{Cl}\end{array}$} \\
\hline & 69 & 89 & 118 & 285 & 33.7 & $4.88(2.81-8.47)$ & & $\rightarrow-$ \\
\hline De Oliveira et al. ${ }^{14)}$ (2014) & 62 & 83 & 10 & 24 & 11.3 & $4.13(1.60-10.69)$ & & - \\
\hline Peralta-Pedrero et al. ${ }^{15)}$ (2006) & 72 & 104 & 136 & 296 & 45.3 & $2.65(1.65-4.26)$ & & $\rightarrow$ \\
\hline Saavedra-González et al. ${ }^{20)}(2015)$ & 12 & 18 & 49 & 202 & 9.6 & $6.24(2.23-17.52)$ & & \\
\hline Total $(95 \% \mathrm{Cl})$ & & 294 & & 807 & 100.0 & $3.72(2.70-5.12)$ & & \\
\hline Total events & 215 & & 313 & & & & & \\
\hline \multirow{2}{*}{\multicolumn{6}{|c|}{ Heterogeneity: chi-square=3.92, df=3 (P=0.27); $I^{2}=24 \%$}} & 0.01 & 0.1 & 11 \\
\hline & & & & & & Protecti & r dep & Risk of d \\
\hline
\end{tabular}

Figure 3. Meta-analysis of the association between family dysfunction and depression. IV, inverse variance; $\mathrm{Cl}$, confidence interval. 


\section{2) Effect of gender on individuals with family dysfunction and risk for depression}

Women with family dysfunction showed a higher prevalence of depressive symptoms during a family medicine consultation regardless of their age; for women between ages 20 and 39 years, the estimated risk is 4.5 times higher than for those with a better perception of family functionality (95\% CI, 2.2-9.4), while for women in the age group between 40 and 59 years, the risk is 1.4 times higher (95\% CI, 0.7-2.9). ${ }^{15)}$ However, family dysfunction is a known risk factor with a greater association among younger individuals. . $^{15,17,22)}$

Motherhood is a social role for women. Mothers of premature infants showed a slightly higher prevalence of depressive symptoms, with a significantly higher association in combination with low family functionality, which is an independent factor related to maternal depressive symptoms. ${ }^{25)}$

\section{3) Effect of social vulnerability on individuals with family dysfunction and risk for depression}

Social vulnerability is determined by various physical, social, economic, and environmental factors that increase one's susceptibility to anticipating, facing, resisting, and recovering from the impacts of a natural hazard. ${ }^{28)}$ Migration is a multifactorial social phenomenon; ${ }^{29)}$ family dysfunction is a risk factor found in populations with this background. A 3-year follow-up of Asian immigrant women revealed a positive screening for depression in those who had family dysfunction. Likewise, patients with impaired family function had higher baseline depression scores compared to those who reported adequate family function. ${ }^{19)}$ Findings among OAs regarding family dysfunction and depression have already been pointed out. Institutionalization is sometimes the result of an OA's inability to meet the specific needs of their life cycle; therefore, it can generally be considered a social vulnerability factor. ${ }^{30)}$ The relationship between social vulnerability and older adulthood increases the probability of presenting with depressive symptoms. ${ }^{14)}$ In North Korean refugees, emotional support and family ties were considered a determining factor linked to the onset of depressive symptoms; similarly, family cohesion has direct and indirect effects on North Korean refugees. ${ }^{23)}$ Family dysfunction in some socially vulnerable populations can be a predictor of severe depression. The prevalence of severe depression among grieving parents who were evaluated 18 months after suffering the loss of their children to the Sichuan earthquake of 2008 was higher among those who reported family dysfunction. ${ }^{24)}$ Through a Pearson's correlation analysis, Cao et al. ${ }^{24)}$ showed that positive family function was significantly related to less severe depression $(\mathrm{r}=-0.46, \mathrm{P}<0.001)$. The more positive the family function, the less severe the depression was as perceived by the grieving parents. ${ }^{24)}$

\section{4) Effect of chronic diseases on individuals with family dysfunction and risk of depression}

Having one or more chronic conditions increases the likelihood of presenting with depressive symptoms. ${ }^{18,27)}$ In people with chronic dis- eases, adequate family support explained the variance in scores obtained on using the scales used for screening for depression. In this sense, adequate family support is considered a protective factor against depressive symptoms, with a greater association found among women than in men. ${ }^{18)}$

The analysis of the study by Wang et al. ${ }^{27)}$ indicates a negative correlation when obtaining lower Family APGAR scores in patients on renal replacement therapy using hemodialysis.

We can affirm that there is an interaction between gender and family satisfaction for the risk of depression in people with diseases.

\section{Strengths and Limitations}

To the best of our knowledge, this is the first systematic review and meta-analysis that shows the relationship between family dysfunction (evaluated through the FACES III and Family APGAR) and depression. A strict protocol was carried out following the international recommendations for conducting systematic reviews and meta-analyses.

The use of screening scales instead of diagnostic scales for depression in some of the studies is one of the limitations of the present work that should be considered in future research. This meta-analysis could not examine relationships with family functionality in other life cycles.

\section{Implications for Practice}

Family dysfunction and depression are multifactorial conditions that require a comprehensive assessment by a primary care physician. It is therefore pertinent to carry out future research involving family functionality to evaluate outcomes regarding depression and other affective symptoms.

\section{Conclusions}

The present study found that there is an association between family dysfunction and depression. It was only possible to find a bidirectional relationship between family function and depression in one of the studies. ${ }^{19)}$ Additionally, being a woman, being a teenager or older adult, having social vulnerability, and having a chronic disease were significantly associated with depression.

The present study highlights the multifactorial nature of the outcome of family dysfunction. This is evident in the subgroup evaluation that was performed for some of the studies; therefore, it is important to mention that when the results are discriminated according to other aspects that allow a better characterization of the individual (gender, age, or social vulnerability), the strength of association can change.

\section{CONFLICT OF INTEREST}

No potential conflict of interest relevant to this article was reported.

\section{ORCID}

Daniel Guerrero-Muñoz: https://orcid.org/0000-0002-1619-5725

Diana Salazar: https://orcid.org/0000-0001-7421-9388 
Victoria Constain: https://orcid.org/0000-0003-1627-6709

Alvaro Perez: https://orcid.org/0000-0001-8841-3798

Carlos Andrés Pineda-Cañar: https://orcid.org/0000-0003-0087-0391

Herney Andrés García-Perdomo: https://orcid.org/0000-0001-6945-8261

\section{REFERENCES}

1. Rakel RE. The family physician. In: Rakel RE, Rakel DP, editors. Textbook of family medicine. 8th ed. Philadelphia (PA): Elsevier Saunders; 2011. p. 3-16.

2. McWhinney IR, Freeman T. Textbook of family medicine. 3rd ed. Oxford: Oxford University Press; 2009.

3. Smilkstein G, Ashworth C, Montano D. Validity and reliability of the Family APGAR as a test of family function. J Fam Pract 1982;15:303-11.

4. Smilkstein G. The Family APGAR: a proposal for a family function test and its use by physicians. J Fam Pract 1978;6:1231-9.

5. Garcia-Caselles P, Miralles R, Arellano M, Torres RM, Aguilera A, PiFigueras M, et al. Validation of a modified version of the Gijon's socialfamilial evaluation scale (SFES): the "Barcelona SFES version", for patients with cognitive impairment. Arch Gerontol Geriatr Suppl 2004;(9):201-6.

6. Olson DH. Circumplex model VII: validation studies and FACES III. Fam Process 1986;25:337-51.

7. Olson DH, Sprenkle DH, Russell CS. Circumplex model of marital and family system: I. Cohesion and adaptability dimensions, family types, and clinical applications. Fam Process 1979;18:3-28.

8. World Health Organization. Depression and other common mental disorders: global health estimates. Geneva: World Health Organization; 2017.

9. American Psychiatric Association. Diagnostic and statistical manual of mental disorders. Arlington (VA): American Psychiatric Publishing; 2013.

10. World Health Organization. The ICD-10 classification of mental and behavioural disorders: clinical descriptions and diagnostic guidelines. Geneva: World Health Organization; 1992.

11. First MB, Reed GM, Hyman SE, Saxena S. The development of the ICD-11 clinical descriptions and diagnostic guidelines for mental and behavioural disorders. World Psychiatry 2015;14:82-90.

12. Slim K, Nini E, Forestier D, Kwiatkowski F, Panis Y, Chipponi J. Methodological index for non-randomized studies (MINORS): development and validation of a new instrument. ANZ J Surg 2003;73:712-6.

13. Higgins JP, Green S. Cochrane handbook for systematic reviews of interventions: version 5.1.0 [Internet]. Oxford: The Cochrane Collaboration; 2011 [cited 2019 May 5]. Available from: https://training.cochrane. org/handbook/archive/v5.1/.

14. De Oliveira SC, dos Santos AA, Pavarini SC. The relationship between depressive symptoms and family functioning in institutionalized elderly. Rev Esc Enferm USP 2014;48:66-72.

15. Peralta-Pedrero ML, Mercado-Castelazo E, Cruz-Avelar A, SanchezMarquez O, Lemus-Rocha R, Martinez-Garcia Mdel C. Depression prevalence and risk factors found in women attended by a family physician. Rev Med Inst Mex Seguro Soc 2006;44:409-14.

16. White J, Shelton KH, Elgar FJ. Prospective associations between the family environment, family cohesion, and psychiatric symptoms among adolescent girls. Child Psychiatry Hum Dev 2014;45:544-54.

17. Lin HC, Tang TC, Yen JY, Ko CH, Huang CF, Liu SC, et al. Depression and its association with self-esteem, family, peer and school factors in a population of 9586 adolescents in southern Taiwan. Psychiatry Clin Neurosci 2008;62:412-20.

18. Nan H, Lee PH, McDowell I, Ni MY, Stewart SM, Lam TH. Depressive symptoms in people with chronic physical conditions: prevalence and risk factors in a Hong Kong community sample. BMC Psychiatry 2012; 12:198.

19. Kim YP, Joh JY, Shin IS. Family function of the families consisting of Asian immigrant women living in South Korea: a 3-year longitudinal study. Asia Pac J Public Health 2015;27:NP2702-11.

20. Saavedra-Gonzalez AG, Garcia-de Leon A, Duarte-Ortuno A, BelloHernandez YE, Infante-Sandoval A. Depression and family functionality in elderly in a family medicine unit from Guayalejo, Tamaulipas, Mexico. Atencion Fam 2016;23:24-8.

21. Souza RA, Desani da Costa G, Yamashita CH, Amendola F, Gaspar JC, Alvarenga MR, et al. Family functioning of elderly with depressive symptoms. Rev Esc Enferm USP 2014;48:469-76.

22. Perez Milena A, Perez Milena R, Martinez Fernandez ML, Leal Helmling FJ, Mesa Gallardo I, Jimenez Pulido I. Family structure and function during adolescence: relationship with social support, tobacco, alcohol and drugs consumption, and psychic discomfort. Aten Primaria 2007;39:61-7.

23. Nam B, Kim JY, DeVylder JE, Song A. Family functioning, resilience, and depression among North Korean refugees. Psychiatry Res 2016; 245:451-7.

24. Cao X, Jiang X, Li X, Lo MC, Li R, Dou X. Perceived family functioning and depression in bereaved parents in China after the 2008 Sichuan earthquake. Arch Psychiatr Nurs 2013;27:204-9.

25. Chang HP, Chen JY, Huang YH, Tyan JY, Yeh CJ, Su PH, et al. Prevalence and factors associated with depressive symptoms in mothers with infants or toddlers. Pediatr Neonatol 2014;55:470-9.

26. Sun L, Sun LN, Sun YH, Yang LS, Wu HY, Zhang DD, et al. Correlations between psychological symptoms and social relationships among medical undergraduates in Anhui Province of China. Int J Psychiatry Med 2011;42:29-47.

27. Wang SY, Zang XY, Liu JD, Cheng M, Shi YX, Zhao Y. Indicators and correlates of psychological disturbance in Chinese patients receiving maintenance hemodialysis: a cross-sectional study. Int Urol Nephrol 2015;47:679-89.

28. Singh SR, Eghdami MR, Singh S. The concept of social vulnerability: a review from disasters perspectives. Int J Interdiscip Multidiscip Stud 2014;1:71-82.

29. Garcia MM, Martinez JL. Immigration and social exclusion. In: Fernandez FV, editor. Social exclusion and the welfare state in Spain. Barcelona: Icaria Editorial; 2006. p. 409-39.

30. Cardona-Arango D, Estrada-Restrepo A, Chavarriaga-Maya LM, Segura-Cardona AM, Ordonez-Molina J, Osorio-Gomez JJ. Dignified social support for elderly institutionalised adults in Medellín, 2008. Rev Salud Publica (Bogota) 2010;12:414-24. 
Appendix 1. Search strategies

\section{MEDLINE (Ovid)}

1. (Famil* adj2 dysfunction*).mp

2. (family dysfunction).mp

3. (Famil* adj2 \$function).mp

4. (family adaptation).mp

5. (famil* adj2 adaptation*).mp

6. (parental adj2 \$function).mp.

7. (famil* adj2 relation*).mp.

8. (famil* adj2 conflict*).mp

9. (famil* adj2 apgar*).mp

10. or/

11. depres*.mp

12. exp depression

13. depressive disorder (Mesh term)

14. Depressive Disorder, Major (Mesh term)

15. Dysthymic Disorder (Mesh term)

16. (Depressive adj2 disorder*).mp

17. or/

18. exp cohort studies

19. (cohort* adj2 stud*).mp

20. exp case-control studies

21. (case* control stud*).mp

22. cross-sectional studies (mesh term)

23. (cross* sectional adj2 stud*).mp

24. or/

25. 10 and 17 and 24

\section{EMBASE}

1. (famil* NEAR/2 dysfunction*):ti,ab)

2. (famil* NEAR/2 function*):ti,ab)

3. (famil* NEAR/2 adaptation):ti,ab)

4. (parental NEAR/2 \$function):ti,ab)

5. (famil* NEAR/2 relation*):ti,ab)

6. (famil* NEAR/2 apgar*):ti,ab)

7. or/

8. 'depression'/exp

9. (depression:ti,ab

10. (dysthymia:ti,ab

11. 'dysthymia'/exp

12. or/

13. 'cohort analysis' /exp

14. (cohort NEXT/2 stud*):ti,ab)

15. 'case control study'/exp

16. case ${ }^{*}$ control:ti,ab

17. 'cross-sectional study'/exp

18. (cross* section*:ti,ab

19. or

20.7 and 12 and 19 